3 Tan E M, Rodnan G P, Garcia I, Moroi Y, Fritzler M J, Peebles C. Diversity of antinuclear antibodies in progressive systemic sclerosis. Arthritis Rheum 1980; 23: 617-25.

4 Giordano M. Classification of progressive systemic sclerosis. International Conference on PSS (scleroderma). Austin, 20-23 October 1981. Arthritis Rheum in press.

5 Giordano M, Valentini G, Ara M, Tirri G, Capelli L, Vatti M. Epidemiology of progressive systemic sclerosis in Italy. International Conference on PSS (scleroderma). Austin, 20-23 October 1981. Arthritis Rheum in press.

\section{Hypothyroidism and calcium pyrophosphate dihydrate deposition disease}

SIR, There has been interest in the possible association between calcium pyrophosphate dihydrate deposition disease (CPPD) and hypothyroidism since the 1975 article by Dorwart and Schumacher. ${ }^{1}$ Recently Alexander et al. ${ }^{2}$ reported the occurrence of hypothyroidism in $10.5 \%$ in 105 patients with CPPD, an incidence higher than expected in an elderly population and higher than in their control groups (osteoarthritis patients and patients requiring acute medical admission). The authors suggested an association between 2 diseases.

Our experience has revealed no obvious association between hypothyroidism and CPPD. We reported the laboratory investigations in 36 CPPD patients and 29 control subjects and found no difference between the mean TSH values (the most sensitive laboratory measurement for hypothyroidism) and an equal incidence of elevated TSH results in the patients and controls. ${ }^{3}$ In a larger compilation McCarty reported no cases of hypothyroidism in 238 CPPD patients. ${ }^{4}$ The 6 of 12 patients reported by Dorwart and Schumacher with calcium pyrophosphate dihydrate crystals present in the synovial fluid were highly selected. All of the patients had florid myxoedema, many were referred to a rheumatologist, and 4 of the 6 patients with CPPD were elderly, ages 72 or older.

Hypothyroidism is frequently associated with musculoskeletal complaints, ${ }^{5}$ and these patients may be overly represented in a rheumatology clinic. The prevalence of both hypothyroidism ${ }^{6}$ and CPPD increases with age, and in any study with older patients both diseases would be expected to be frequently encountered.

Before we accept hypothyroidism as an associated disease with CPPD we should await a study comparing the prevalence of CPPD in hypothyroid patients versus an age and sex-matched euthyroid control group.

MICHAEL H. ELLMAN

Division of Rheumatology,

Michael Reese Hospital and Medical Center,

2929 South Ellis Avenue,

Chicago, Illinois 60616, USA

\section{References}

1 Dorwart B B, Schumacher H R. Joint effusions, chondrocalcinosis and other rhe umatic manifestations in hypothyroidism: a clinicopathologic study. Am J Med 1975; 59: 780-90.

2 Alexander G M, Dieppe P A, Doherty M, et al. Pyrophosphate arthropathy: a study of metabolic associations and laboratory data. Ann Rheum Dis 1982; 41: 377-81.

3 Ellman M H, Brown N L, Porat A P. Laboratory investigations in pseudogout patients and controls. J Rheumatol 1980; 7: 77-81.

4 McCarty D J. Pseudogout: articular chondrocalcinosis. In: Hollander J L, McCarty D J, eds. Arthritis and Allied Conditions. 8th ed. Philadelphia: Lea and Febiger, 1972: 1140-60.

5 Bland J H, Frymoyer J W. Rhe umatic syndromes of myxedema. $N$ Engl J Med 1970; 282: 1171-8.

6 Blum M. Thyroid function and disease in the elderly. Hosp Pract 1981; 16: 105-16.

\section{Age-related blood changes in hip osteoarthritis patients: a possible indicator of bone quality}

SIR, Rapin and Lagier, in your correspondence columns, ${ }^{1}$ report a significantly high mean serum albumin, calciumo and 25-hydroxycholecalciferol levels in patients with hip osteoarthritis compared with patients with femoral nects fractures and similar aged controls. These findings do not? correspond with our experience. ${ }^{2-4}$ Our present data on calcium metabolism in women with primary osteoarthrosis and primary osteoporosis are summarised in Table 1 . The only difference between osteoporosis and osteoarthrosis we could find was a higher calciuria, and a higher calcium/creatinine ratio in a morning spot urine while fasting, in patients with osteoporosis. The latter findings point to a more negative bone balance due to increased bone resorption in osteoporosis.

The discordance between our findings and those of Rapin and Lagier can be explained by several factors. Firstly, our patients were all women; the osteoarthrosis patients all had Heberden nodes plus arthrosis at other joints, mostly on hips or knees; and the osteoporosis patients all suffered from at least one vertebral collapse, which is a failure of trabecular bone. The sex of the patients of Rapin and Lagier is not mentioned. The Osteoarthrosis cases were a mixed group of patients with coxarthrosis, which can be the result of different diseases (primary or secondary osteoarthrosis), and the osteoporosis cases had femoral neck fracture, which results from failure of cortical bone. Secondly, the difference in serum calcium levels in the study of Rapin and Lagier is due to a higher serum albumin level in the osteoarthrosis group. In our study there was no difference in serum total protein, as there was no difference in serum calcium, phosphate, and alkaline phosphatase activity. Thirdly, 25-hydroxycholecalciferol is not the physiologically active vitamin D metabolite and is subject to seasonal variation ${ }^{5}$ and sunlight exposure. 1-25-dihydroxycholecalciferol on the other hand, which 